\title{
Effect of Temperature on Photonic Band Gaps in Semiconductor-Based One-Dimensional Photonic Crystal
}

\author{
J. V. Malik, ${ }^{1}$ K. D. Jindal, ${ }^{2}$ Vinay Kumar, ${ }^{2}$ Vipin Kumar, ${ }^{3}$ Arun Kumar, \\ Kh. S. Singh, ${ }^{3}$ and T. P. Singh ${ }^{1}$ \\ ${ }^{1}$ Department of Physics, Janta Vedic College, Baraut 250611, India \\ ${ }^{2}$ Department of Physics, Shri Venkateshwara University, Gajraula, J. P. Nagar, India \\ ${ }^{3}$ Department of Physics, Digamber Jain College, Baraut 250611, India \\ ${ }^{4}$ AITEM, Amity University, Noida, India
}

Correspondence should be addressed to J. V. Malik; jvmalik@yahoo.com

Received 15 March 2013; Accepted 20 July 2013

Academic Editor: Saulius Juodkazis

Copyright (c) 2013 J. V. Malik et al. This is an open access article distributed under the Creative Commons Attribution License, which permits unrestricted use, distribution, and reproduction in any medium, provided the original work is properly cited.

\begin{abstract}
The effect of the temperature and angle of incidence on the photonic band gap (PBG) for semiconductor-based photonic crystals has been investigated. The refractive index of semiconductor layers is taken as a function of temperature and wavelength. Three structures have been analyzed by choosing a semiconductor material for one of the two materials in a bilayer structure. The semiconductor material is taken to be $\mathrm{ZnS}, \mathrm{Si}$, and $\mathrm{Ge}$ with air in first, second, and third structures respectively. The shifting of band gaps with temperature is more pronounced in the third structure than in the first two structures because the change in the refractive index of Ge layers with temperature is more than the change of refractive index of both $\mathrm{ZnS}$ and Si layers with temperature. The propagation characteristics of the proposed structures are analyzed by transfer matrix method.
\end{abstract}

\section{Introduction}

Since the pioneering works of Yablonovitch [1] and John [2], studies on the electromagnetic properties of photonic crystals which are artificial structures with periodically modulated dielectric constants have been attracting a great deal of interest among the researchers. Photonic crystals that exhibit electromagnetic stop bands or photonic band gaps (PBGs) have received considerable attention over the last two decades for the study of their fundamental physical properties as well as for their potential applications in many optoelectronic devices [3-13]. It was observed that periodic modulation of the dielectric functions significantly modifies the spectral properties of the electromagnetic waves. The electromagnetic transmission and/or reflection spectra in such structures are/is characterized by the presence of allowed and forbidden photonic energy bands similar to the electronic band structure of periodic potentials. For this reason, such a new class of artificial optical material with periodic dielectric modulation is known as photonic band gap (PBG) material [14]. Fundamental optical properties like band structure, reflectance, group velocity, and rate of spontaneous emission, can be controlled effectively by changing the spatial distribution of the dielectric function $[5,6]$.

A 1D PC structure has many interesting applications such as dielectric reflecting mirrors, low-loss waveguides, optical switches, filters, optical limiters. It has also been demonstrated theoretically and experimentally that 1D PCs have absolute omnidirectional PBGs [15-19].

In addition to the existence of wide photonic band gaps in some properly designed PCs, the feature of a tunability of PBGs in PCs attracts the attention of investigators in recent years. PBGs can be tuned by means of some external agents. For instance, band gaps can be shifted by changing the operating temperature, and such method of tuning may be called temperature tuning or simply T-tuning [20]. A superconductor/dielectric PC belongs to this type of photonic crystals. This happens because of the temperature-dependent 


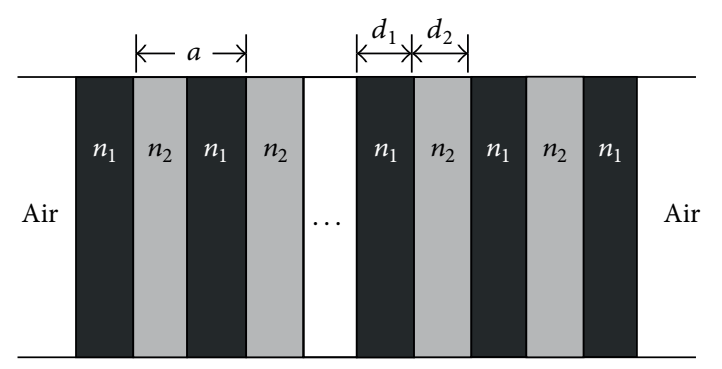

FIGURE 1: Schematic diagram of 1D photonic crystal structure.

London perturbation length in the superconducting materials [21-24].

However, in earlier reports on one-dimensional PCs based on dielectric media studied by the researchers the dielectric property of the constituent layers was considered to be independent of the temperature and nondispersive. In this paper, we consider semiconductor media as one of the constituents of a one-dimensional photonic crystal in which the dielectric property of semiconductors depends not only on temperature but also on wavelength. Here we consider the three multilayer systems, namely, ZnS/air, Si/air, and Ge/air. The refractive index of air is independent of temperature and wavelength. But the refractive indices of semiconductor layers are taken as a function of both temperature and wavelength $[25,26]$. Therefore, this study may be considered to be physically more realistic.

\section{Theoretical Analysis}

The schematic representation of one-dimensional photonic crystal is represented in Figure 1.

We consider air $/(A B)^{N} A$ /air structure in which $A$ and $B$ represent the high and low refractive index materials. To compute the transmission spectrum, we employ the transfer matrix method (TMM) [27]. In this method, the transfer matrix for each layer can be written as

$$
M_{j}=D_{j} P_{j} D_{j}^{-1}
$$

where $j$ stands for $A$ or $B$ layers and $D_{j}$ and $P_{j}$ are called the dynamical matrix and the propagation matrix, respectively. The dynamical matrix is given by the following equations:

$$
\begin{gathered}
D_{j}=\left(\begin{array}{cc}
1 & 1 \\
n_{j} \cos \theta_{j} & -n_{j} \cos \theta_{j}
\end{array}\right) \text { for TE mode of polarization, } \\
D_{j}=\left(\begin{array}{cc}
\cos \theta_{j} & \cos \theta_{j} \\
n_{j} & -n_{j}
\end{array}\right) \text { for TM mode of polarization. }
\end{gathered}
$$

Also, the propagation matrix $P_{j}$ can be defined as

$$
P_{j}=\left(\begin{array}{cc}
e^{i \delta_{j}} & 0 \\
0 & e^{-i \delta_{j}}
\end{array}\right)
$$

where the phase is written as

$$
\delta_{j}=\frac{2 \pi d_{j}}{\lambda} n_{j} \cos \theta_{j}
$$

The transfer matrix for the structure embedded in air, the transfer matrix for the $\operatorname{air} /(A B)^{N} A$ /air structure, can be written as

$$
M=\left(\begin{array}{ll}
M_{11} & M_{12} \\
M_{21} & M_{22}
\end{array}\right)=D_{0}^{-1}\left(M_{A} M_{B}\right)^{N} M_{A} D_{0},
$$

where $D_{0}$ is called the dynamical matrix for air.

The reflection and transmission coefficients in terms of the matrix elements given in (5) can be written as

$$
\begin{aligned}
& r=\frac{\left(M_{11}+q_{f} M_{12}\right) q_{i}-\left(M_{21}+q_{f} M_{22}\right)}{\left(M_{11}+q_{f} M_{12}\right) q_{i}+\left(M_{21}+q_{f} M_{22}\right)} \\
& t=\frac{2 q_{i}}{\left(M_{11}+q_{f} M_{12}\right) q_{i}+\left(M_{21}+q_{f} M_{22}\right)}
\end{aligned}
$$

where $q_{i, f}=n_{i, f} \cos \theta_{j, f}$ for TE wave and $q_{i, f}=\left(\cos \theta_{j, f}\right) / n_{i, f}$ for TM wave, where the subscripts $i$ and $f$ correspond to the quantities, respectively, in the medium of incidence and the medium of emergence. Whereas, the reflectance and transmittance of the structure is given by

$$
R=|r|^{2}, \quad T=|t|^{2} .
$$

\section{Proposed Structures and Structural Parameters}

We choose $\mathrm{ZnS}, \mathrm{Si}$, and $\mathrm{Ge}$ for the material $A$, air for material $B$, and $N=10$ in Figure 1. So, there will be three proposed structures: [air/(ZnS/air) ${ }^{10} \mathrm{ZnS} /$ air], [air/(Si/air) ${ }^{10} \mathrm{Si} /$ air] and [air/(Ge/air) ${ }^{10} \mathrm{Ge} /$ air]. We take $\mathrm{ZnS}, \mathrm{Si}$, and $\mathrm{Ge}$ as the high refractive index materials and air as the low refractive index material. The geometrical parameters are so chosen that the thicknesses of high and low refractive index materials are the same at $300 \mathrm{~K}$ temperature; that is, $d_{1}=d_{2}$ for all structures. We confine our study in the temperature range from 300 to $700 \mathrm{~K}$ only so that the three structures can be compared.

3.1. Structure I: [air/(ZnS/air $)^{10} \mathrm{ZnS} /$ air]. The refractive index of $\mathrm{ZnS}$ layer is taken as a function of both wavelength and temperature. The thermal expansion coefficient and melting point for $\mathrm{ZnS}$ layers are taken to be $6.1 \times 10^{-6} / \mathrm{K}$ and $1830 \mathrm{~K}$, respectively [28]. The refractive index of $\mathrm{ZnS}$ in the ranges $1200-14000 \mathrm{~nm}$ and $293-700 \mathrm{~K}$ can be expressed as a function of both the wavelength and temperature as [25]

$$
n^{2}(\lambda, T)=\varepsilon_{1}(T)+\frac{A(T)}{\lambda^{2}-\lambda_{1}^{2}}+\frac{B(T)}{\left(\lambda / \lambda_{2}\right)^{2}-1},
$$


where

$$
\begin{aligned}
\lambda_{1}= & 0.23979+4.841 \times 10^{-5} T_{1}, \\
\lambda_{2}=36.525 & +4.75 \times 10^{-3} T_{1}, \quad T_{1}=T-293, \\
\varepsilon_{1}(T)= & 8.34096+1.29107 \times 10^{-3} T_{1} \\
& +4.68388 \times 10^{-7} T_{1}^{2} \\
& -1.31683 \times 10^{-9} T_{1}^{3} \\
& -6.64356 \times 10^{-12} T_{1}^{4}, \\
A(T)= & 0.14540+1.13319 \times 10^{-5} T_{1} \\
& +1.05932 \times 10^{-8} T_{1}^{2} \\
& +1.06004 \times 10^{-10} T_{1}^{3} \\
& +2.27671 \times 10^{-13} T_{1}^{4}, \\
B(T)= & 3.23924+1.096 \times 10^{-3} T_{1} \\
& +4.20092 \times 10^{-7} T_{1}{ }^{2} \\
& +1.1135 \times 10^{-9} T_{1}^{3} \\
& +7.2992 \times 10^{-12} T_{1}^{4} .
\end{aligned}
$$

3.2. Structure II: [air/(Si/air $)^{10} \mathrm{Si} /$ air]. The thermal expansion coefficient and melting point for Si layer are taken to be 2.6 $\times 10^{-6} / \mathrm{K}$ and $1685 \mathrm{~K}$, respectively [28]. The refractive index of air is independent of temperature and wavelength. But the refractive index of silicon layer is taken as a function of both wavelength and temperature. The refractive index of silicon $(\mathrm{Si})$ in the ranges $1200-14000 \mathrm{~nm}$ and $20-1600 \mathrm{~K}$ is represented as [26]

$$
\begin{aligned}
n^{2}(\lambda, T)= & \varepsilon(T)+\frac{e^{-3 \Delta L(T) / L_{293}}}{\lambda^{2}} \\
& \times\left(0.8948+4.3977 \times 10^{-4} T+7.3835 \times 10^{-8} T^{2}\right),
\end{aligned}
$$

where

$$
\begin{aligned}
\mathcal{E}(T)= & 11.4445+2.7739 \times 10^{-4} T \\
& +1.7050 \times 10^{-6} T^{2} \\
& -8.1347 \times 10^{-10} T^{3}
\end{aligned}
$$

$$
\begin{aligned}
\frac{\Delta L(T)}{L_{293}}= & -0.00071+1.887 \times 10^{-6} T \\
& +1.934 \times 10^{-9} T^{2} \\
& -4.554 \times 10^{-13} T^{3}
\end{aligned}
$$

for $20 \mathrm{~K} \leq T \leq 293 \mathrm{~K}$,

$$
\begin{aligned}
\frac{\Delta L(T)}{L_{293}}= & -0.00021-4.149 \times 10^{-7} T \\
& -4.620 \times 10^{-10} T^{2} \\
& +1.482 \times 10^{-11} T^{3}
\end{aligned}
$$

for $293 \mathrm{~K} \leq \mathrm{T} \leq 1600 \mathrm{~K}$.

3.3. Structure III: [air/(Ge/air $)^{10}$ Ge/air]. The thermal expansion coefficient and melting point for Ge layer are taken to be $6.9 \times 10^{-6} / \mathrm{K}$ and $1210 \mathrm{~K}$, respectively [28]. The refractive index of Ge layer is taken as a function of both wavelength and temperature. The refractive index of Ge in the ranges 1200$14000 \mathrm{~nm}$ and $293-1000 \mathrm{~K}$ can be expressed as a function of both the wavelength and temperature as [26]

$$
\begin{aligned}
n^{2}(\lambda, T)= & \varepsilon(T)+\frac{e^{-3 \Delta L(T) / L_{293}}}{\lambda^{2}} \\
& \times\left(2.5381+1.8260 \times 10^{-3} T+2.8888 \times 10^{-6} T^{2}\right),
\end{aligned}
$$

where

$$
\begin{aligned}
\varepsilon(T)= & 15.2892+1.4549 \times 10^{-3} T \\
& +3.5078 \times 10^{-6} T^{2} \\
& -1.2071 \times 10^{-9} T^{3}, \\
\frac{\Delta L(T)}{L_{293}}= & 5.790 \times 10^{-5}(T-293) \\
& +1.768 \times 10^{-9}(T-293)^{2} \\
& -4.562 \times 10^{-13}(T-293)^{3}
\end{aligned}
$$

for $293 \mathrm{~K} \leq T \leq 1000 \mathrm{~K}$.

\section{Results and Discussion}

4.1. Structure I: $\left[a i r /(Z n S / a i r)^{10} Z n S / a i r\right]$. The plot of the refractive index of $\mathrm{ZnS}$ as the function of wavelength and temperature is shown in Figure 2(a). From this figure, it is clear that the refractive index of $\mathrm{ZnS}$ layers increases with temperature. Therefore refractive index contrast increases with temperature. So the photonic band gaps can be shifted as a function of temperature. The variation of reflectance with temperature at different temperatures is shown in Figure 3 


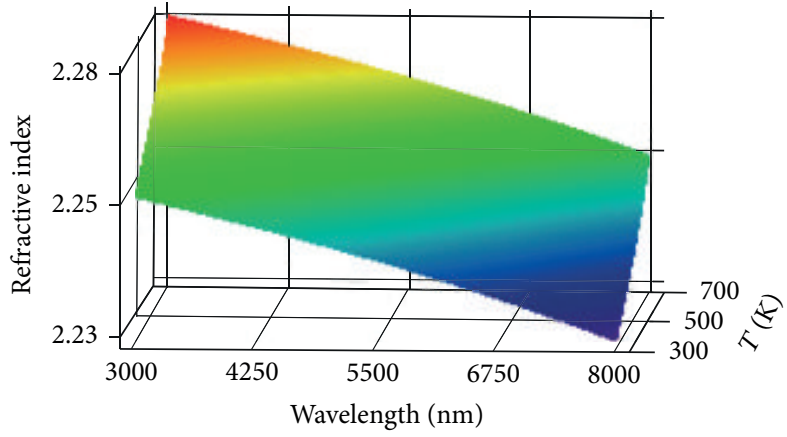

(a)

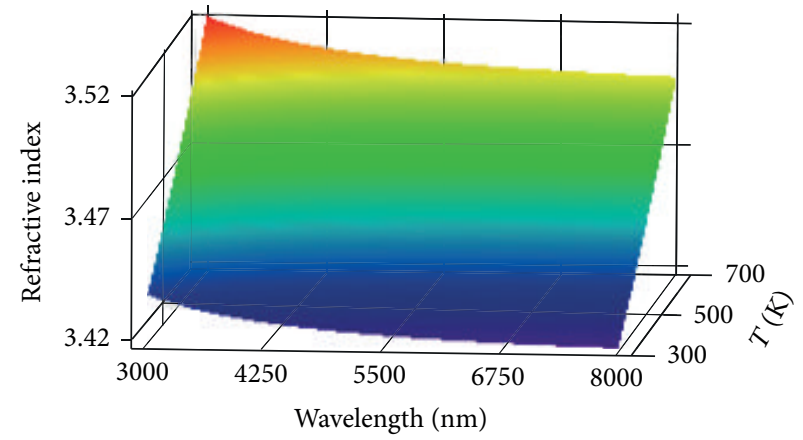

(b)

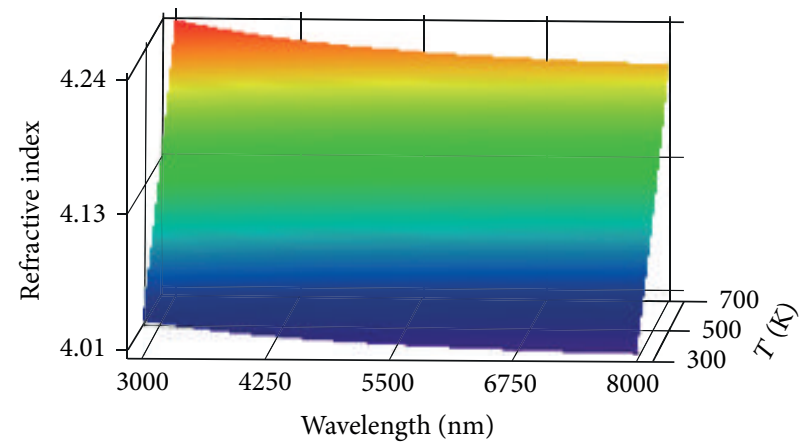

(c)

FiguRE 2: Variation of refractive index with wavelength and temperature, (a) ZnS, (b) Si, and (c) Ge.

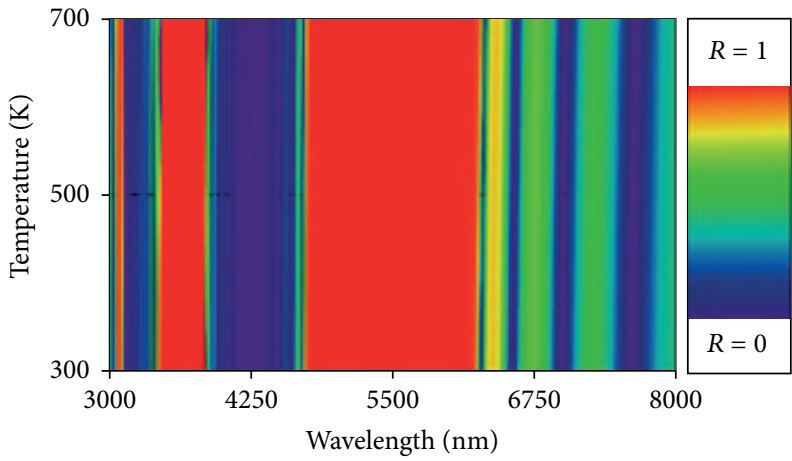

(a) $\theta=0^{\circ}$

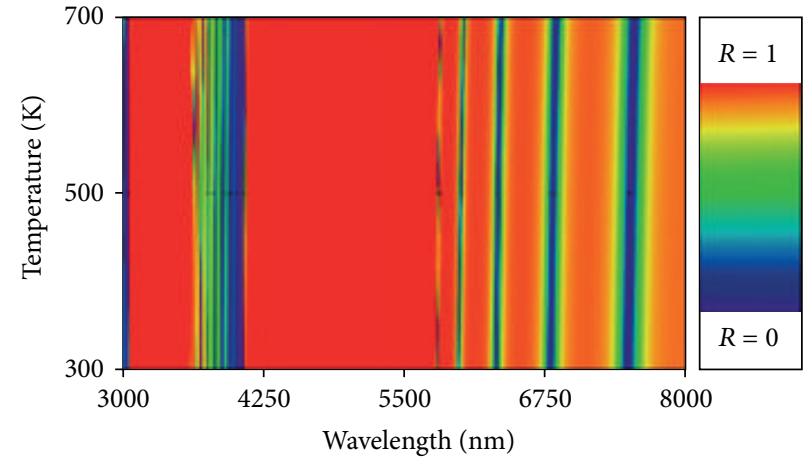

(b) $\theta=85^{\circ}$

FIGURE 3: Reflectance with temperature for ZnS/air.

and corresponding data tabulated in Table 1 in which the complete photonic band gaps (CPBGs) and corresponding central wavelengths for band gaps are given for two angles of incidence (i.e., $0^{\circ}$ and $85^{\circ}$ ) and at two different temperatures (i.e., $300 \mathrm{~K}$ and $700 \mathrm{~K}$ ).

Two complete band gaps appear in the reflection spectra. From these figures and table, it is clear that as we increase the temperature (at constant angle of incidence) the band gaps shift slightly towards the longer wavelength region. The shifting of band gaps with temperature is slight because the refractive index of $\mathrm{ZnS}$ layers changes slowly with temperature. On the other hand, as we increase the angle of incidence (at constant temperature) the band gaps shift towards the shorter wavelength region, but the PBG width 


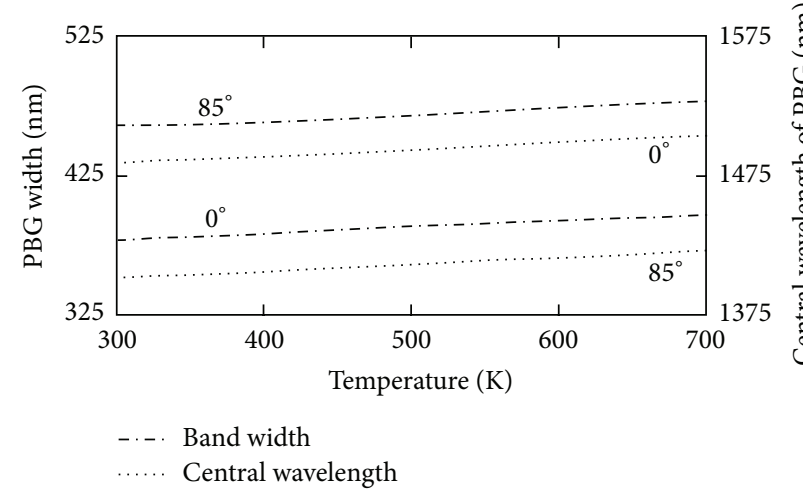

(a)

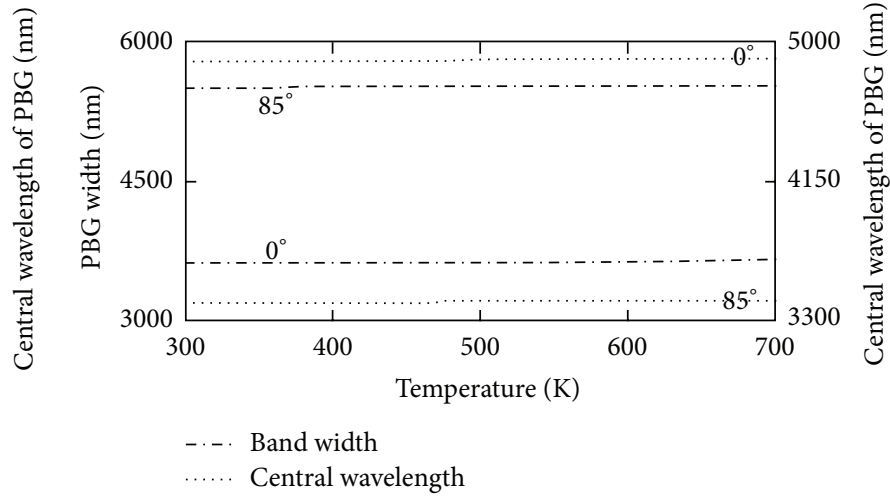

(b)

FIGURE 4: Variation of PBG band width and central wavelength of PBG with temperature for ZnS/air.

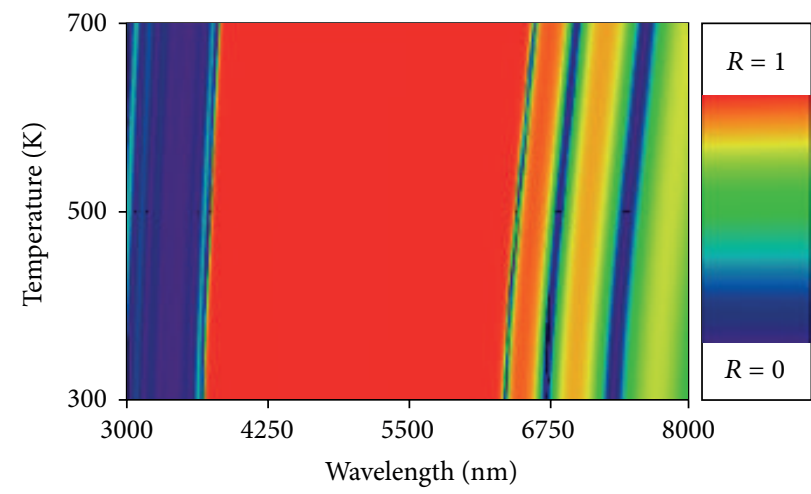

(a) $\theta=0^{\circ}$

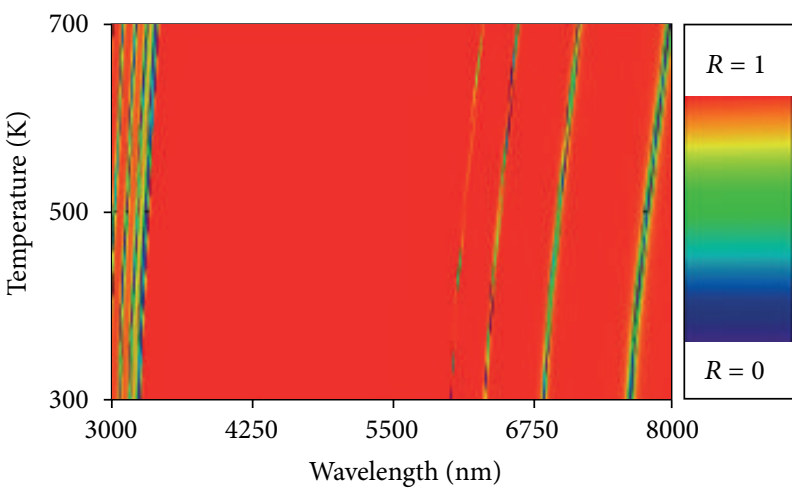

(b) $\theta=85^{\circ}$

FIGURE 5: Reflectance with temperature for Si/air.

TABLE 1: PBG width and central wavelength of BAND-GAP at different angles of incidence and temperatures for $\mathrm{ZnS} /$ air multilayer system.

\begin{tabular}{|c|c|c|c|c|}
\hline & \multicolumn{4}{|c|}{ Temperature } \\
\hline & \multicolumn{2}{|c|}{$300 \mathrm{~K}$} & \multicolumn{2}{|c|}{$700 \mathrm{~K}$} \\
\hline & \multicolumn{4}{|c|}{ Angle of incidence } \\
\hline & $0^{\circ}$ & $85^{\circ}$ & $0^{\circ}$ & $85^{\circ}$ \\
\hline \multirow[t]{2}{*}{ CPBG width (nm) } & 380 & 460 & 398 & 479 \\
\hline & 1402 & 1485 & 1421 & 1504 \\
\hline \multirow[t]{2}{*}{ Central wavelength (nm) } & 3626 & 3405 & 3645 & 3428 \\
\hline & 5500 & 4876 & 5522 & 4897 \\
\hline
\end{tabular}

increases. The regions with $R \approx 1$ are corresponding to the complete photonic band gaps. The variation of PBG and band width with temperature is shown in Figure 4 . Figure 4 shows the variation of PBGs and central wavelength of these PBGs with angle of incidence. From this figure it is clear that if we increase the temperature, the band width and central wavelengths of these PBGs increase, but the central wavelength shifts towards the shorter wavelength region as we increase the angle of incidence.

4.2. Structure II: [air/(Si/air $)^{10}$ Si/air]. The plot of the refractive index of Si as the function of wavelength and temperature is shown in Figure 2(b). From this figure, it is clear that the refractive index of $\mathrm{ZnS}$ layers increases with temperature. Therefore refractive index contrast increases with temperature. In this case the change in refractive contrast is more than that in the case of structure I. The variation of reflectance with temperature at different temperatures is shown in Figure 5, and corresponding data are tabulated in Table 2.

Only one complete band gap (CPBG) appears in the reflection spectra for this case. From these figures and table, it is clear that as we increase the temperature (at constant 


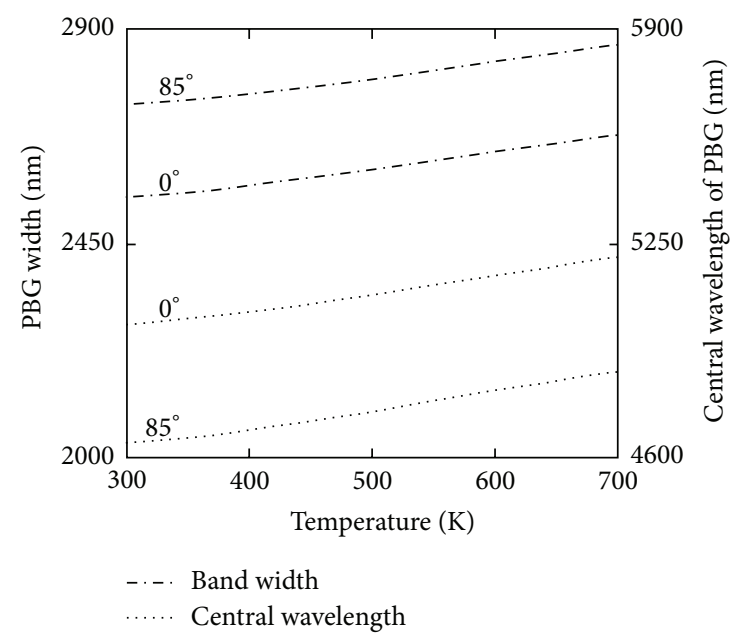

FIGURE 6: Variation of PBG band width and central wavelength of PBG with temperature for Si/air.

TABLE 2: PBG width and central wavelength of BAND-GAP at different angles of incidence and temperatures for Si/air multilayer system.

\begin{tabular}{|c|c|c|c|c|}
\hline & \multicolumn{4}{|c|}{ Temperature } \\
\hline & \multicolumn{2}{|c|}{$300 \mathrm{~K}$} & \multicolumn{2}{|c|}{$700 \mathrm{~K}$} \\
\hline & \multicolumn{4}{|c|}{ Angle of incidence } \\
\hline & $0^{\circ}$ & $85^{\circ}$ & $0^{\circ}$ & $85^{\circ}$ \\
\hline CPBG width (nm) & 2548 & 2742 & 2679 & 2868 \\
\hline Central wavelength (nm) & 5007 & 4646 & 5208.5 & 4865 \\
\hline
\end{tabular}

angle of incidence) the band gaps shift slightly towards the longer wavelength region. The shifting of band gaps with temperature is more than that of structure I because the change in the refractive index of Si layers with temperature is more than the change in the refractive index of $\mathrm{ZnS}$ with temperature. On the other hand, as we increase the angle of incidence (at constant temperature) the band gaps shift towards the shorter wavelength region, but the PBG width increases. The variation of PBG and band width with temperature is shown in Figure 6. This figure shows the variation of PBGs and central wavelength of these PBGs with angle of incidence. From this figure it is clear that if we increase the temperature the band width and center of these PBGs increase, but the central wavelength shifts towards the shorter wavelength region for large angle of incidence.

4.3. Structure III: [air/(Ge/air $)^{10}$ Ge/air]. The plot of the refractive index of $\mathrm{Ge}$ as the function of wavelength and temperature is shown in Figure 2(c). From this figure, it is clear that the refractive index of Ge layers increases with temperature. Therefore refractive index contrast increases
TABLE 3: PBG width and central wavelength of BAND-GAP at different angles of incidence and temperatures for Ge/air multilayer system.

\begin{tabular}{|c|c|c|c|c|}
\hline & \multicolumn{4}{|c|}{ Temperature } \\
\hline & \multicolumn{2}{|c|}{$300 \mathrm{~K}$} & \multicolumn{2}{|c|}{$700 \mathrm{~K}$} \\
\hline & \multicolumn{4}{|c|}{ Angle of incidence } \\
\hline & $0^{\circ}$ & $85^{\circ}$ & $0^{\circ}$ & $85^{\circ}$ \\
\hline \multirow[t]{2}{*}{ CPBG width (nm) } & 602 & 783 & 764 & 941 \\
\hline & 2018 & 2922 & 2189 & 3104 \\
\hline \multirow[t]{2}{*}{ Central wavelength (nm) } & 3590 & 3410 & 3710 & 3534 \\
\hline & 6205 & 5911 & 6583 & 6279 \\
\hline
\end{tabular}

with temperature. The variation of reflectance with temperature at different temperatures is shown in Figure 7, and corresponding data are tabulated in Table 3.

Two complete band gaps appear in the reflection spectra. From these figures and table, it is clear that as we increase the temperature (at constant angle of incidence) the band gaps shift slightly towards the longer wavelength region. The shifting of band gaps with temperature is more pronounced than in the first two cases, because the rate of change in the refractive index of Ge layers with temperature is more than the rate of change of refractive index of both $\mathrm{ZnS}$ and Si layers with temperature. On the other hand, as we increase the angle of incidence (at constant temperature) the band gaps shift towards the shorter wavelength region, but the PBG width increases. The variation of complete PBGs and band widths with temperature is shown in Figure 8. This Figure shows the variation of PBGs and central wavelength of these PBGs with angle of incidence. From this figure it is clear that if we increase the temperature the band width and center of these PBG increase but the central wavelength shifts towards the shorter wavelength region for large angle of incidence.

The shifting behavior for all cases can be explained by using the phase equation (4) [29]. According to this phase equation, as $n(\lambda, T)$ increases with temperature, the wavelength must increase accordingly to keep the phase $\delta$ unchanged. The shifting with angle of incidence is due to the cosine function on the right-hand side. As we increase the angle of incidence, the value of the cosine function on the right-hand side decreases. So the wavelength must decrease accordingly to keep the phase $\delta$ unchanged. This behavior is helpful in tuning the PBG width and central wavelength of PBG.

\section{Conclusion}

The effect of the temperature and angle of incidence on the PBG for semiconductor-based photonic crystals has been investigated. The refractive indices of semiconductor layers are taken as a function of both temperature and wavelength. So, the simulation can be used for the wide range of temperature as well as wavelength. Therefore, this study 


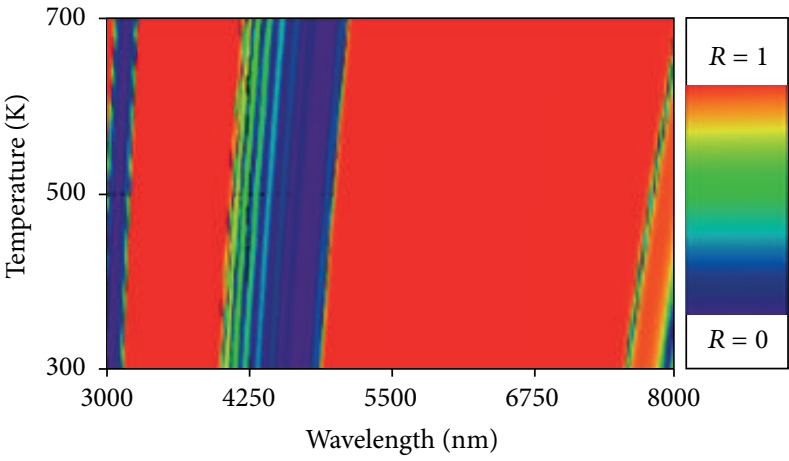

(a) $\theta=0^{\circ}$

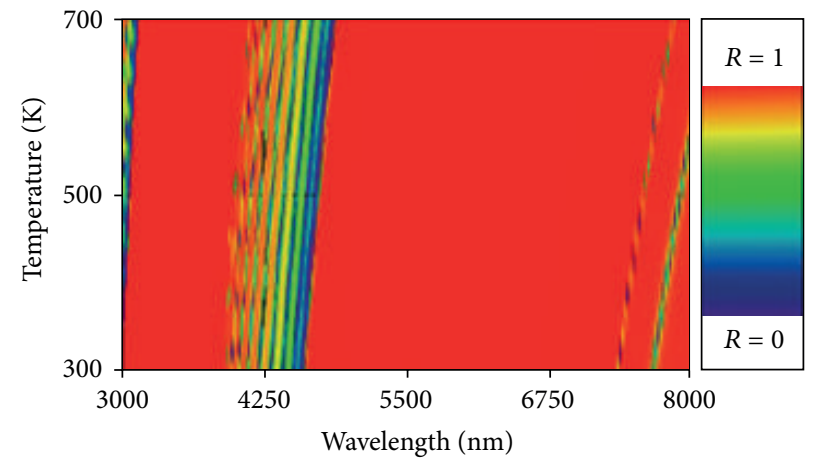

(b) $\theta=85^{\circ}$

Figure 7: Reflectance with temperature for Ge/air.

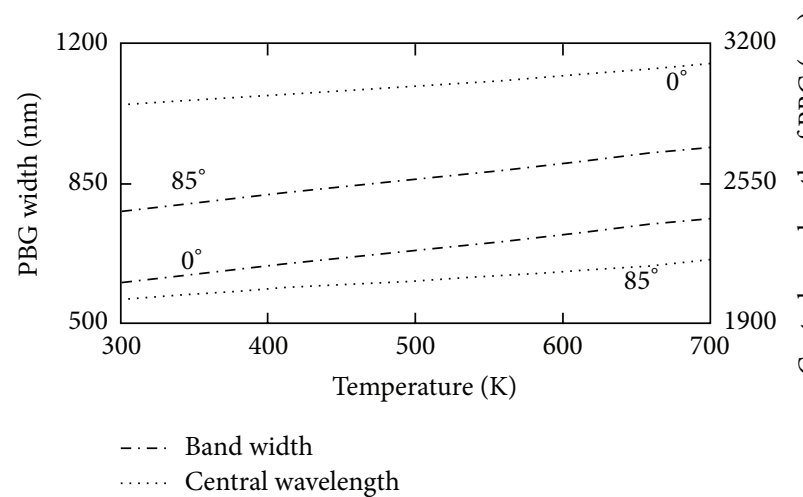

(a)

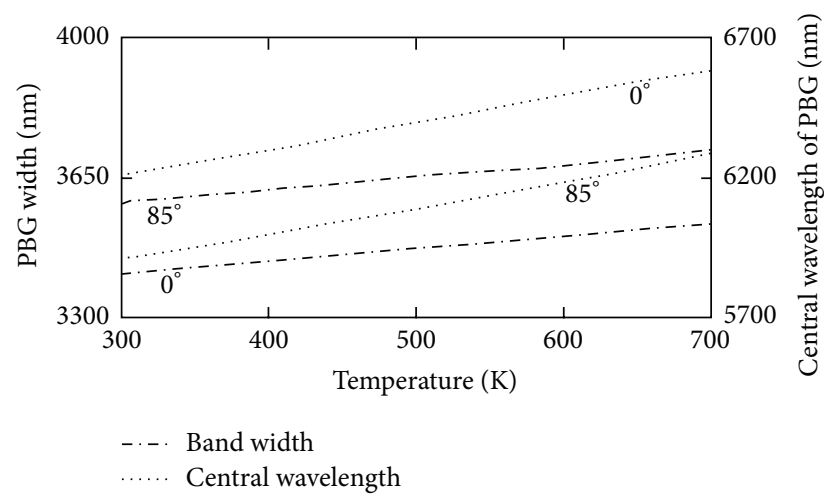

(b)

FIGURE 8: Variation of PBG band width and central wavelength of PBG with temperature for Ge/air.

may be considered to be physically more realistic. We can use the proposed structures as temperature sensing device, narrow band optical filter, wavelength division demultiplexer, and tunable omnidirectional reflector and in many optical systems.

\section{Acknowledgment}

One of the authors, Vipin Kumar, wishes to express sincere gratitude to Professor S. P. Ojha for his valuable suggestions and critical comments.

\section{References}

[1] E. Yablonovitch, "Inhibited spontaneous emission in solid-state physics and electronics," Physical Review Letters, vol. 58, no. 20, pp. 2059-2062, 1987.

[2] S. John, "Strong localization of photons in certain disordered dielectric superlattices," Physical Review Letters, vol. 58, no. 23, pp. 2486-2489, 1987.
[3] K. M. Ho, C. T. Chan, and C. M. Soukoulis, "Existence of a photonic gap in periodic dielectric structures," Physical Review Letters, vol. 65, no. 25, pp. 3152-3155, 1990.

[4] J. D. Joannopoulos, P. R. Villeneuve, and S. Fan, "Photonic crystals: putting a new twist on light," Nature, vol. 386, no. 6621, pp. 143-149, 1997.

[5] J. A. M. Rojas, J. Alpuente, J. Piñeiro, and R. Sánchez, "Rigorous full vectorial analysis of electromagnetic wave propagation in ID inhomogeneous media," Progress in Electromagnetics Research, vol. 63, pp. 89-105, 2006.

[6] E. Yablonovitch and T. J. Gmitter, "Photonic band structure: the face-centered-cubic case," Physical Review Letters, vol. 63, no. 18, pp. 1950-1953, 1989.

[7] J. D. Joannopoulos, R. D. Meade, and J. N. Winn, Photonic Crystals: Molding the Flow of Light, Princeton University Press, Princeton, NJ, USA, 1995.

[8] E. Burstein and C. Weisbuch, Confined Electron and Photon: New Physics and Applications, Plenum Press, New York, NY, USA, 1995. 
[9] V. Kumar, K. S. Singh, and S. P. Ojha, "Band structure, reflection properties and abnormal behaviour of one-dimensional plasma photonic crystals," Progress in Electromagnetics Research M, vol. 9, pp. 227-241, 2009.

[10] A. Kumar, V. Kumar, B. Suthar, M. Ojha, Kh. S. Singh, and S. P. Ojha, "Trapping of light in nonlinear 1D photonic crystal," Photonics Technology Letters, vol. 25, no. 3, p. 279, 2013.

[11] A. Kumar, V. Kumar, B. Suthar, A. Bhargava, Kh. S. Singh, and S. P. Ojha, "Wide range temperature sensors based on onedimensional photonic crystal with a single defect," International Journal of Microwave Science and Technology, vol. 2012, Article ID 182793, 5 pages, 2012.

[12] A. Kumar, B. Suthar, V. Kumar, Kh. S. Singh, and A. Bhargava, "Tunable wavelength demultiplexer for DWDM application using 1-d photonic crystal," Progress in Electromagnetics Research Letters, vol. 33, pp. 27-35, 2012.

[13] V. Kumar, B. Suthar, A. Kumar et al., "Wave transmission in dispersive Si-based one dimensional photonic crystal," Optics and Photonics Journal, vol. 2, no. 3A, pp. 237-241, 2012.

[14] C. Soukoulis, Photonic Band Gap Materials, Kluwer Academic, Dordrecht, The Netherlands, 1996.

[15] J. P. Dowling, "Mirror on the wall: you're omnidirectional after all?” Science, vol. 282, no. 5395, pp. 1841-1842, 1998.

[16] E. Yablonovitch, "Engineered omnidirectional externalreflectivity spectra from one-dimensional layered interference filters," Optics Letters, vol. 23, no. 21, pp. 1648-1649, 1998.

[17] D. N. Chigrin, A. V. Lavrinenko, D. A. Yarotsky, and S. V. Gaponenko, "Observation of total omnidirectional reflection from a one-dimensional dielectric lattice," Applied Physics A, vol. 68, no. 1, pp. 25-28, 1999.

[18] B. Suthar, V. Kumar, K. S. Singh, and A. Bhargava, "Tuning of photonic band gaps in one dimensional chalcogenide based photonic crystal," Optics Communications, vol. 285, no. 6, pp. 1505-1509, 2012.

[19] V. Kumar, K. S. Singh, S. K. Singh, and S. P. Ojha, "Broadening of omnidirectional photonic band gap in Si-based one dimensional photonic crystals," Progress In Electromagnetics Research M, vol. 14, pp. 101-111, 2010.

[20] V. Kumar, B. Suthar, A. Kumar, Kh. S. Singh, and A. Bhargva, "Effect of temperature on photonic band gap in dispersive sibased one dimensional photonic crystal," Physica B, vol. 416, pp. 106-109, 2013.

[21] O. L. Berman, Y. E. Lozovik, S. L. Eiderman, and R. D. Coalson, "Superconducting photonic crystals: numerical calculations of the band structure," Physical Review B, vol. 74, no. 9, Article ID 092505, 2006.

[22] H. Takeda and K. Yoshino, "Tunable photonic band schemes in two-dimensional photonic crystals composed of copper oxide high-temperature superconductors," Physical Review B, vol. 67, no. 24, Article ID 245109, 6 pages, 2003.

[23] W.-H. Lin, C.-J. Wu, T.-J. Yang, and S.-J. Chang, “Terahertz multichanneled filter in a superconducting photonic crystal," Optics Express, vol. 18, no. 26, pp. 27155-27166, 2010.

[24] M.-S. Chen, C.-J. Wu, and T.-J. Yang, "Investigation of optical properties in near-zero-permittivity operation range for a superconducting photonic crystal," Applied Physics A, vol. 104, no. 3, pp. 913-919, 2011.

[25] H. H. Li, "Refractive index of silicon and germanium and its wavelength and temperature derivatives," Journal of Physical and Chemical Reference Data, vol. 9, no. 3, p. 561, 1980.
[26] H. H. Li, "Refractive index of $\mathrm{ZnS}$ and its wavelength and temperature derivatives," Journal of Physical and Chemical Reference Data, vol. 13, no. 1, p. 103, 1984.

[27] P. Yeh, Optical Waves in Layered Media, John Wiley and Sons, New York, NY, USA, 1988.

[28] G. Ghosh, Handbook of Thermo-Optic Coefficients of Optical Materials with Applications, Academic Press, New York, NY, USA, 1985.

[29] B. Suthar, V. Kumar, A. Kumar, Kh. S. Singh, and A. Bhargava, "Thermal expansion of photonic band gap for one-dimensional photonic crystal," Progress in Electromagnetics Research Letters, vol. 32, pp. 81-90, 2012. 

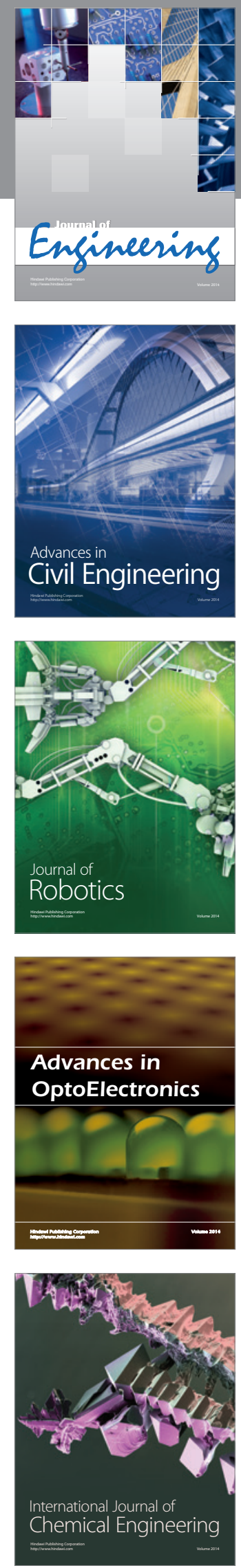

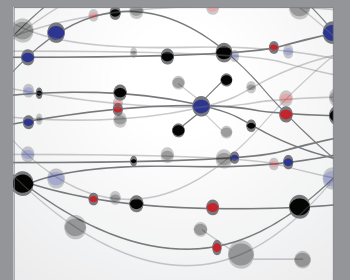

The Scientific World Journal
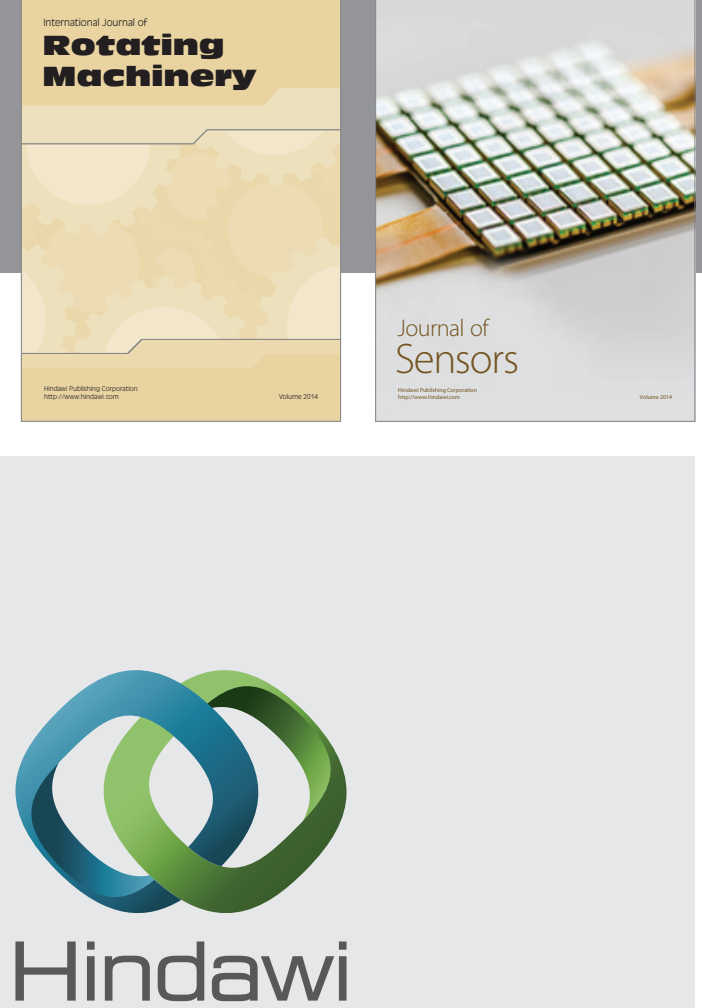

Submit your manuscripts at http://www.hindawi.com
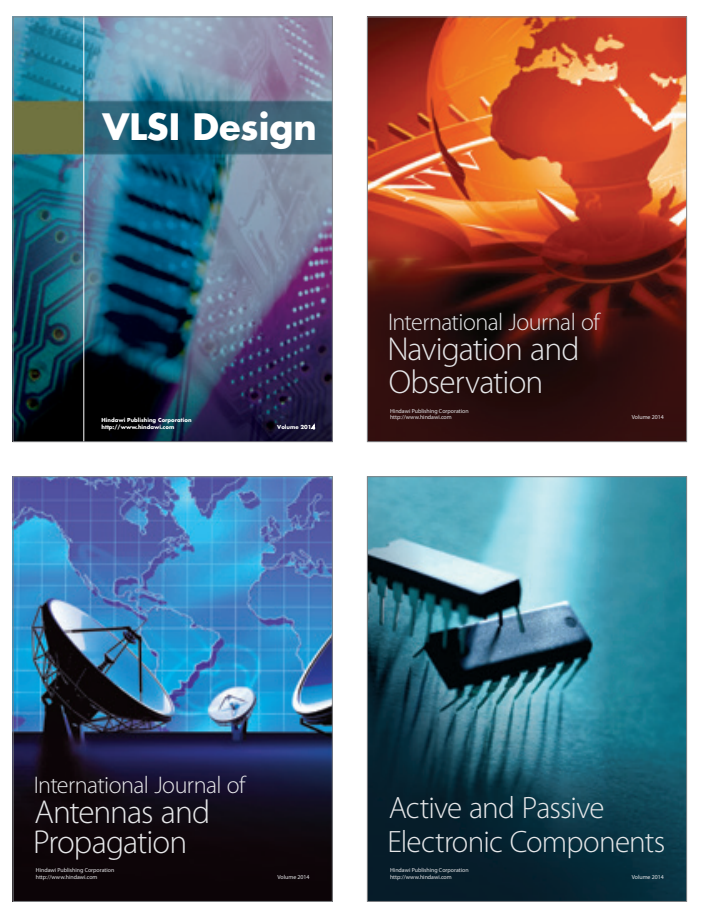
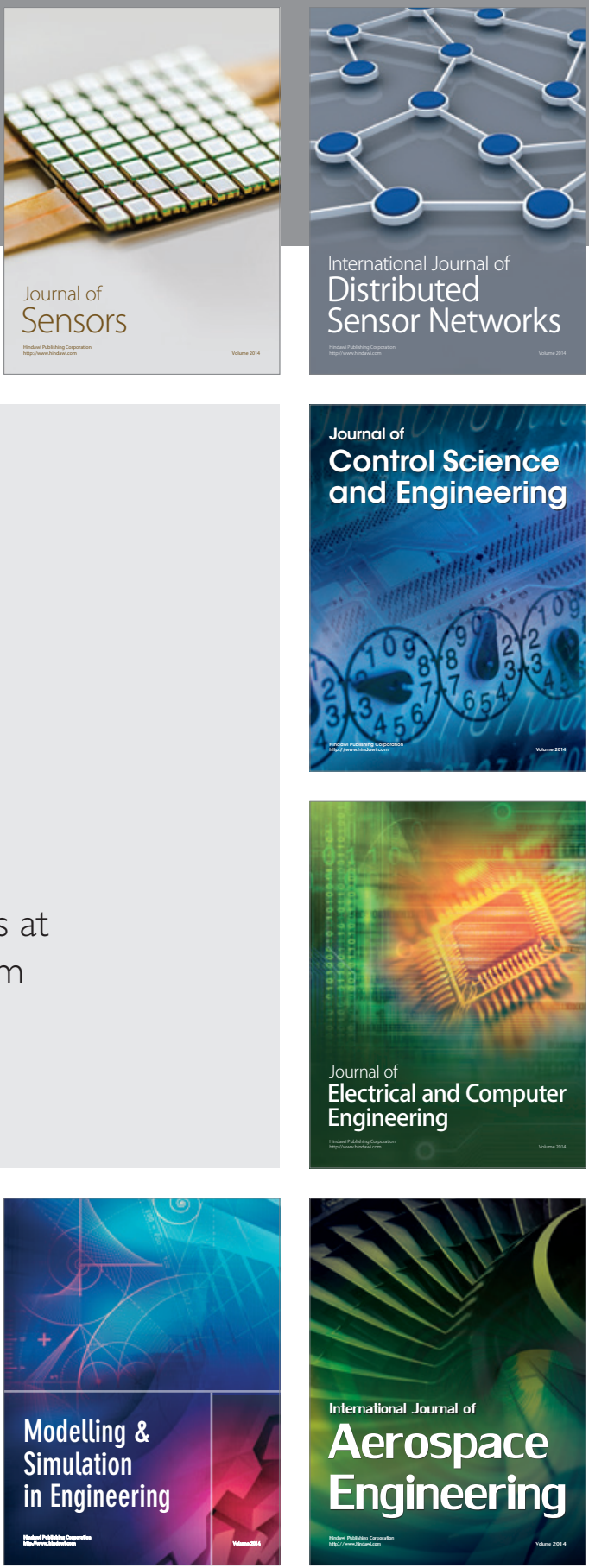

Journal of

Control Science

and Engineering
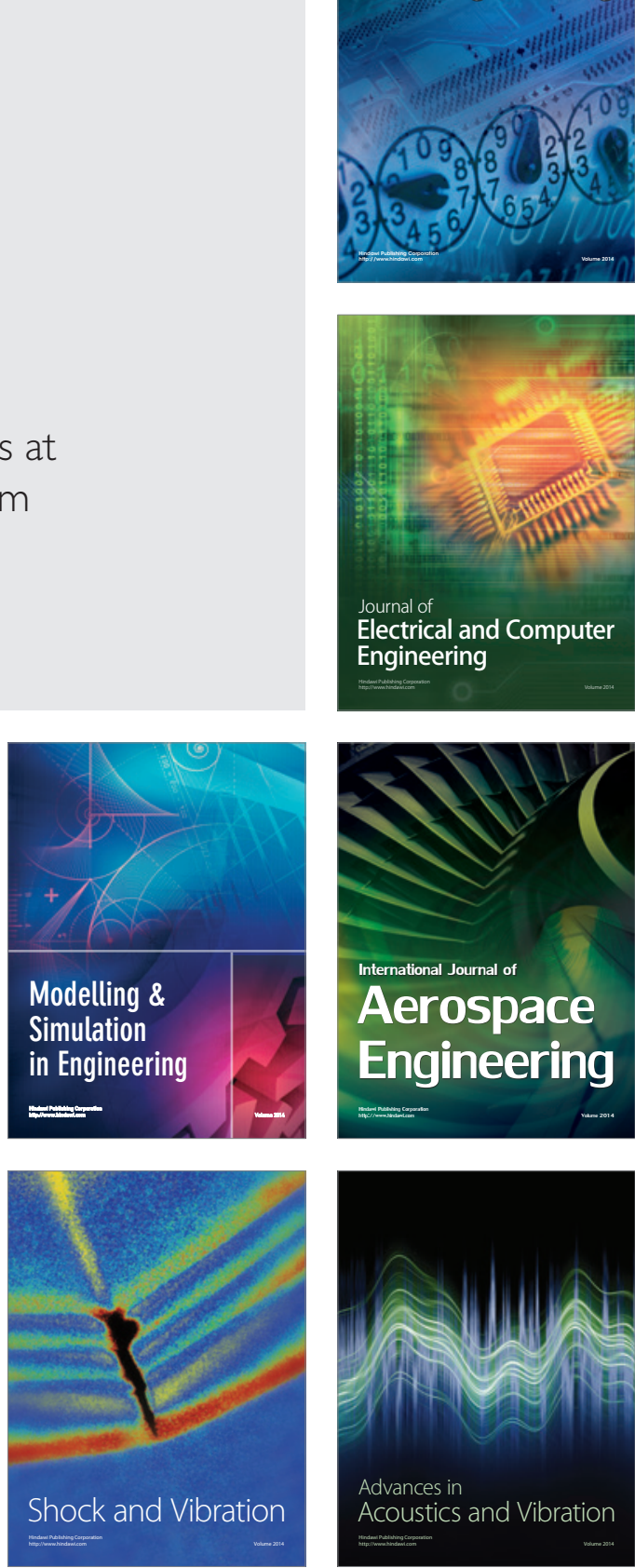